\title{
Mechanisms for Adjusting Interaural Time Differences to Achieve Binaural Coincidence Detection
}

\author{
Armin H. Seidl, Edwin W Rubel, and David M. Harris \\ Virginia Merrill Bloedel Hearing Research Center, Department of Otolaryngology-Head and Neck Surgery, University of Washington, Seattle, Washington \\ 98195-7923
}

Understanding binaural perception requires detailed analyses of the neural circuitry responsible for the computation of interaural time differences (ITDs). In the avian brainstem, this circuit consists of internal axonal delay lines innervating an array of coincidence detector neurons that encode external ITDs. Nucleus magnocellularis (NM) neurons project to the dorsal dendritic field of the ipsilateral nucleus laminaris (NL) and to the ventral field of the contralateral NL. Contralateral-projecting axons form a delay line system along a band of NL neurons. Binaural acoustic signals in the form of phase-locked action potentials from NM cells arrive at NL and establish a topographic map of sound source location along the azimuth. These pathways are assumed to represent a circuit similar to the Jeffress model of sound localization, establishing a place code along an isofrequency contour of NL. Three-dimensional measurements of axon lengths reveal major discrepancies with the current model; the temporal offset based on conduction length alone makes encoding of physiological ITDs impossible. However, axon diameter and distances between Nodes of Ranvier also influence signal propagation times along an axon. Our measurements of these parameters reveal that diameter and internode distance can compensate for the temporal offset inferred from axon lengths alone. Together with other recent studies, these unexpected results should inspire new thinking on the cellular biology, evolution, and plasticity of the circuitry underlying low-frequency sound localization in both birds and mammals.

\section{Introduction}

Binaural processing of acoustic signals is essential for localizing sound and extracting signals in a noisy environment. Lowfrequency sounds are localized by interaural differences in the arrival times of sound created by a spatial separation of the ears [interaural time differences (ITDs)]. ITDs are thought to be computed by a neural mechanism similar to that proposed by Jeffress (1948), where external differences of sound arrival times at the ears are represented along internal delay lines.

In the avian auditory brainstem, a modified Jeffress model is represented by nucleus magnocellularis (NM) and nucleus laminaris (NL) (see Fig. 1A). Neurons in NM receive monaural input from the ipsilateral acoustic sensory epithelium of birds via the auditory nerve (AN). NM axons bifurcate and send bilateral projections to neurons in NL (see Fig. $1 A$ ). The ipsilateral NM axon provides a simultaneous input to the dorsal dendrites of an isofrequency array of NL neurons (Hyson et al., 1994) and by traveling an extended looped trajectory putatively equalizes conduction times with the contralateral pathway (Jhaveri and Morest, 1982;

\footnotetext{
Received July 16, 2009; revised 0ct. 30, 2009; accepted Nov. 5, 2009.

This work was supported by National Institutes of Health-National Institute on Deafness and Other Communication Disorders Grants DC003829, DC004661, and DC008042. We thank Glen MCDonald and Dale Cunningham for technical assistance. Dale Cunningham was pivotal in preparing the semithin and ultrathin sections. We also thank Rock Levinson for suggesting the caspr antibody. We are grateful to Yuan Wang, Kathryn Tabor, Melissa Caras, Yong Lu, R. Michael Burger, Jason Tait Sanchez, and Daniel T. Kashima for discussion and criticism on the manuscript. We thank the anonymous reviewers for useful comments and constructive criticism. Parts of this study were presented previously (Harris et al., 2008).

Correspondence should be addressed to Edwin W Rubel, Virginia Merrill Bloedel Hearing Research Center, University of Washington, Box 357923, Seattle, WA 98195-7923. E-mail: rubel@uw.edu.

DOI:10.1523/JNEUROSCI.3464-09.2010

Copyright $\odot 2010$ the authors $\quad 0270-6474 / 10 / 300070-11 \$ 15.00 / 0$
}

Young and Rubel, 1983) (see Fig. 1B). Coincidence detection for 0 ITD in the NL has recently been shown in vivo (Köppl and Carr, 2008). The contralateral axons cross the midline and travel on the ventral side along an isofrequency band of NL neurons. Terminal arbors branch off the main axon in serial order, thereby creating a physical delay line (Young and Rubel, 1983).

NL neurons function as an array of coincidence detectors (Carr and Konishi, 1990; Joseph and Hyson, 1993; Hyson et al., 1994; Reyes et al., 1996; Kuba et al., 2002). NL neurons distributed along the delay line respond maximally to particular ITDs, thus establishing a neural map of sound source location in the azimuth. When a stimulus comes from straight ahead (ITD $=0$ ), the specific location on the map receiving equivalent delay from each ear responds maximally. Sound source locations generating ITDs different from 0 are encoded by an array of neurons along internal delay lines that compensate for the different external arrival times (see Fig. $1 B$ ).

We analyzed the anatomy of the NM-NL circuit to determine what mechanisms are used to compensate for external delays and its fit for the model described above. Our three-dimensional (3-D) measurements of axon length do not comply with the proposed model in its simple form (Overholt et al., 1992). The contralateral axon is on average $>1600 \mu \mathrm{m}$ longer than its ipsilateral counterpart, rendering encoding of physiological ITDs based on conduction length alone impossible; coincident binaural inputs at NL would only be created by ITDs larger than $180 \mu \mathrm{s}$, hence outside the physiological range (Hyson et al., 1994; Hyson, 2005). We also measured axon diameter and distances between Nodes of Ranvier in different segments of the NM axon. These parameters vary across the NM axonal arbor and act to slow transmission of 


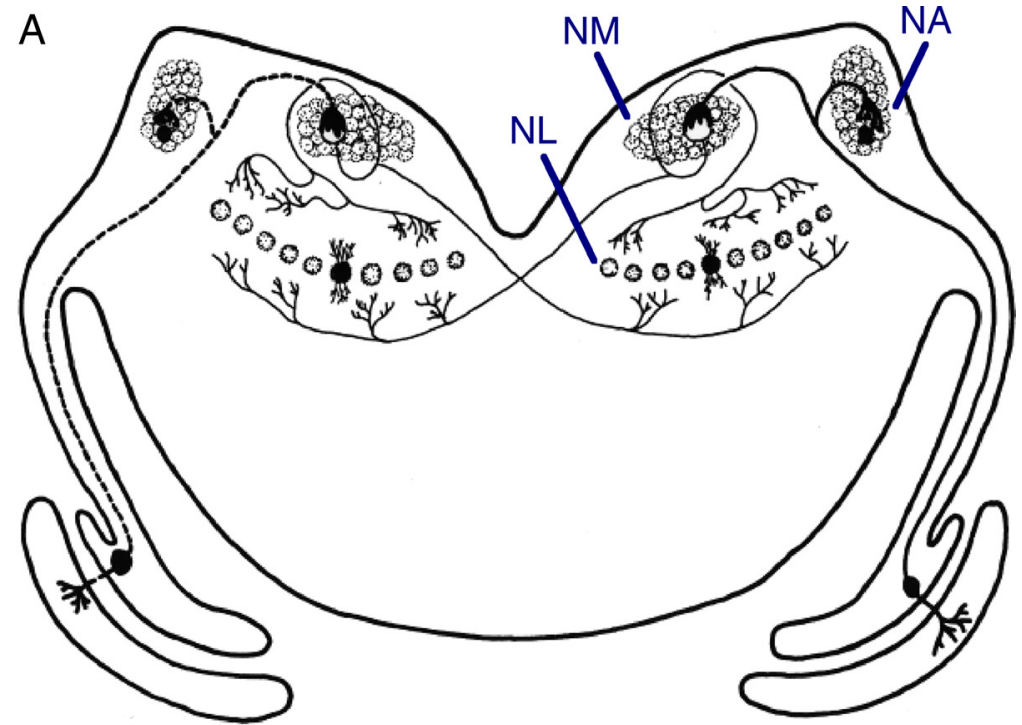

B
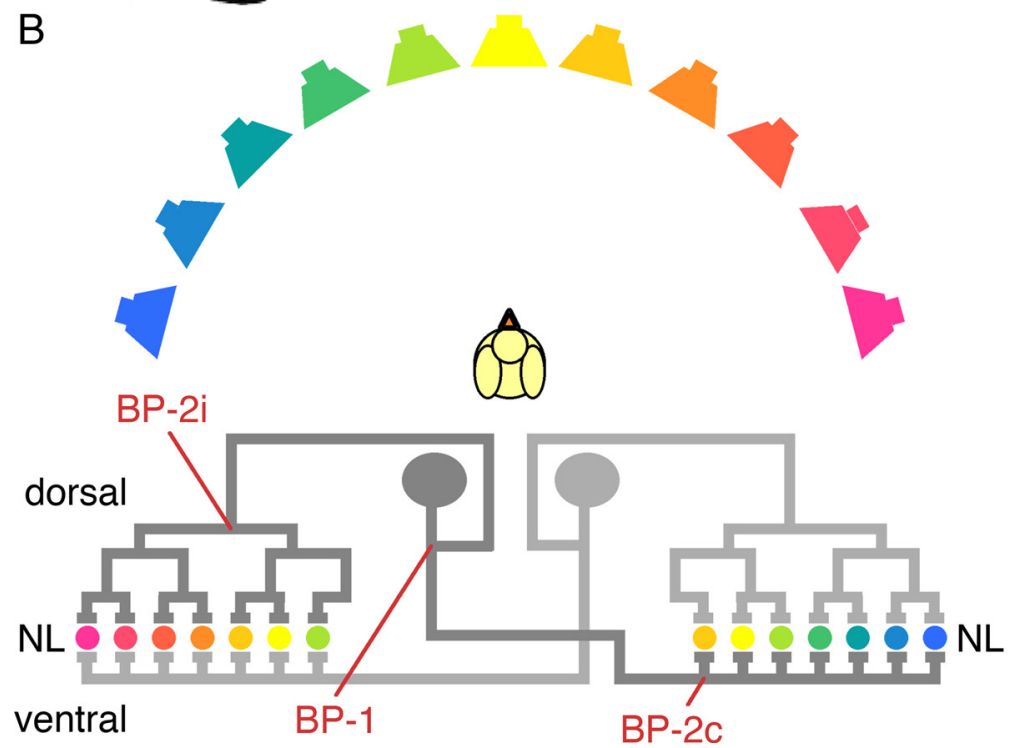

Figure 1. Chicken sound localization circuit. A, Anatomic diagram of the sound localization circuit in the chicken auditory brainstem (coronal view). NM receives acoustic information via the auditory nerve alongside NA. NM projects to the ventral side of the contralateral NL and the dorsal side of the ipsilateral NL. The contralateral NM axon forms a delay line parallel to an isofrequency contour of NL, with increasing delays from medial to lateral. $\boldsymbol{B}$, Schematic representation of the modified Jeffress model embodied by the chicken auditory brainstem. Color-coded speakers represent different sound source positions along the azimuth. NL cells are coincidence detectors and respond best to the sound source of the same color. The ipsilateral loop putatively equalizes conduction times with the contralateral axon. The ipsilateral axon terminals provide an isochronic input to the dorsal dendrites of NL, while the contralateral input is systematically delayed from medial to lateral. Note how sound from straight ahead is encoded by neurons near the medial edge of NL and how most of the NL is tuned to contralateral leading sounds. BP, Branching point; BP-1, BP-2i, and BP2c indicate the first and second branching points ipsilaterally and contralaterally, respectively.

the acoustically evoked signal in the ipsilateral branch of the NM axon compared to the longer contralateral branch. It appears that these determinates of conduction velocity are adjusted to compensate for the axon length offset. Our results provide implications for the ITD detection mechanism not only in birds, but also in mammals, as recent studies have challenged the status quo of its proposed mechanism, i.e., its fit for the Jeffress model (McAlpine et al., 2001; Brand et al., 2002).

\section{Materials and Methods}

Animals. Over 50 white leghorn chickens (Gallus gallus) of varied postnatal ages were studied. The day of hatching was considered to be post- natal day $0(\mathrm{P} 0)$. The University of Washington Institutional Animal Care and Use Committee approved all procedures.

3-D reconstruction of auditory brainstem $n u-$ clei. Chickens of different ages (P0, P1, P14, P15, P27) were deeply anesthetized (urethane 1 $\mathrm{mg} / \mathrm{kg}$, ketamine $20 \mathrm{mg} / \mathrm{kg}$ ) and perfused intracardially with $0.9 \%$ saline plus $0.4 \%$ heparin, followed by $4 \%$ paraformaldehyde in phosphate buffer (PFA) plus 1\% methylene blue. Adequate perfusion was achieved when the beak and feet turned dark blue. The entire head was further fixed by immersion in 4\% PFA for $24 \mathrm{~h}$ at room temperature $\left(\mathrm{RT} ; 21^{\circ} \mathrm{C}\right)$ and then decalcified in RDO (Apex Engineering Products Cooperation) for 3-4 h. Specimens were passed through a series of sucrose gradients over $48 \mathrm{~h}$ for cryoprotection and then blocked in the desired plane of sectioning. Then entire heads were put into an embedding medium (Tissue-Tek O.C.T. Compound, Sakura Finetek) under vacuum for 2-3 h to remove air from the middle ear cavities.

Digitized image stacks of aligned and stained serial sections undistorted by histological manipulation were made with a "photomicrotome" (Harris et al., 1990), a freezing microtome with a digital camera (Nikon D200, 10.5 Megapixels, Nikon) attached, focused on the cutting plane. The embedded chicken head was positioned on the stage of the microtome, covered with embedding medium and packed in dry ice. Frozen sections were serially cut at $22 \mu \mathrm{m}$. Before each frozen section was cut, an image of the surface of the block was captured and saved online. In this way, the whole brain was imaged in situ along with the skull. The histological sections containing the nucleus angularis (NA), AN, NM, and NL were then stained with cresyl violet to aid in identifying nuclear boundaries and structures during subsequent digital segmentation.

The acquired image stacks were contrastenhanced in Photoshop (Adobe) and then loaded into Amira software (Mercury Software) for reconstruction of the three-dimensional anatomy. The volume and length of the resulting Amira 3-D models were measured using ImageJ (National Institute of Mental Health, Bethesda, MD).

3-D tracing of individual NM-axons. Thick slices $(1000 \mu \mathrm{m})$ containing the auditory brainstem of 12 newly hatched chickens (P0/1) were prepared in ice-cold artificial CSF (ACSF) (Sorensen and Rubel, 2006) using a Vibratome (Vibratome Series 1000, The Vibratome Company) and a sapphire knife (Leica Microsystems). One slice per animal was harvested and immediately incubated in oxygenated ACSF at RT for $45 \mathrm{~min}$. With the caudal side of the slice facing upward, NM was visualized and small clusters of NM cells were filled with Alexa Fluor568 dextran $(20 \mathrm{~mm}$ in sterile $0.9 \%$ saline; Invitrogen) by electroporation through a glass pipette positioned in NM [tip diameter 1-2 $\mu \mathrm{m}$, adapted from Haas et al. (2001), Burger et al. (2005), and Sorensen and Rubel (2006)]. After electroporation, the slice was incubated for another $4-5 \mathrm{~h}$ at RT in oxygenated ACSF. The slices were subsequently fixed in $4 \% \mathrm{PFA}$ for $10 \mathrm{~min}$, rinsed in phosphate buffered saline (PBS), dehydrated in a series of ethanol steps and put into a clearing solution [3:5 mixture of Benzyl benzoate and Methyl salicylate (MacDonald and Rubel, 2008)]. Filled cells and axons could be imaged in their entirety in these $1000 \mu \mathrm{m}$ tissue slabs using confocal microscopy (Fluoview 1000, Olympus). Brain 
slices containing filled cells and axon extensions to both ipsi- and contralateral NL were imaged by recording several Z-stacks of tiffimages. The resulting Z-stacks were loaded into Neurolucida (MBF Bioscience, Williston, VT) and the cell and its projections traced in 3-D over every Z-stack containing labeled ipsi- and contralateral projections to $\mathrm{NL}$. The resulting 3-D trace was analyzed and measured in Neurolucida Explorer (MBF Bioscience).

Measurement of individual dorsal and ventral $N M$ terminal fields on NL. Individual dorsal and ventral NM terminal fields were measured in the 3-D tracings we described in the previous paragraph. On the ipsilateral side, we measured the axon length from the second branch point of the ipsilateral axon (BP-2i) (Fig. 1B) to the respective axon ending. On the contralateral side, we measured axon length from the point where the axon segment branched off the main axon to the axonal ending, i.e., we did not include the delay line in the measurements. All measurements were done in Neurolucida Explorer.

Additionally, previously published material of Young and Rubel $(1983,1986)$ was used to measure the axonal terminal trees of NM neurons onto NL cells. Briefly, horseradish peroxidase (HRP) tracer injections were made in the crossed dorsal cochlear tract (XDCT) of chick embryos at embryonic day 17 (E17). NM cells and fibers were filled with HRP and both ipsiand contralateral terminal trees of NM axons onto NL were identified. Camera lucida drawings of HRP-labeled terminal fields of single NM axons were scanned and saved as digital files. All measurements were calibrated by scale bars depicted in respective images. Individual segments of axonal terminal trees to the ventral and dorsal of NL were traced and the lengths measured in Image $(\mathrm{NIH}, \mathrm{Be}-$ thesda, Maryland). When fibers extended over several histological sections (50 $\mu \mathrm{m}$ section thickness), the Pythagorean theorem was applied to adjust the measured length to compensate for foreshortening.

In Figure $8 A$ a schematic of the terminal tree demonstrates the pattern of terminal field branching of NM terminates onto NL dendrites. Branch Point 1 (BP-1) is the first bifurcation point. BP-2i is the second ipsilateral bifurcation point; $\mathrm{BP}-2 \mathrm{c}$ is the second contralateral bifurcation point (Fig. $1 B$ ).

Plastic embedding for determination of axon diameter. Electron microscopy of ultrathin (0.1 $\mu \mathrm{m})$ and light microscopy of semithin (1-2 $\mu \mathrm{m})$ sagittal sections of chicken brainstem were used to measure axon diameter at the midline of the XDCT and through areas lateral of the midline that contain NM axons as they approach the ipsi- and contralateral NL. Chickens (P0/1) were deeply anesthetized with an intraperitoneal injection of pentobarbital and prepared for cardiac perfusion. Initial washout was made with $0.1 \mathrm{M}$ cacodylate buffer $(\mathrm{CB}, \mathrm{pH} 7.4)$ containing $0.001 \% \mathrm{CaCl}_{2}$ and $4 \mathrm{U} / \mathrm{ml}$ heparin. This was followed by perfusion with $2 \% \mathrm{PFA}, 2.5 \%$ glutaraldehyde in CB. Chicks were decapitated, skulls opened and heads immersed in the same fixative for $1 \mathrm{~h}$ on a platform rotator and then overnight in
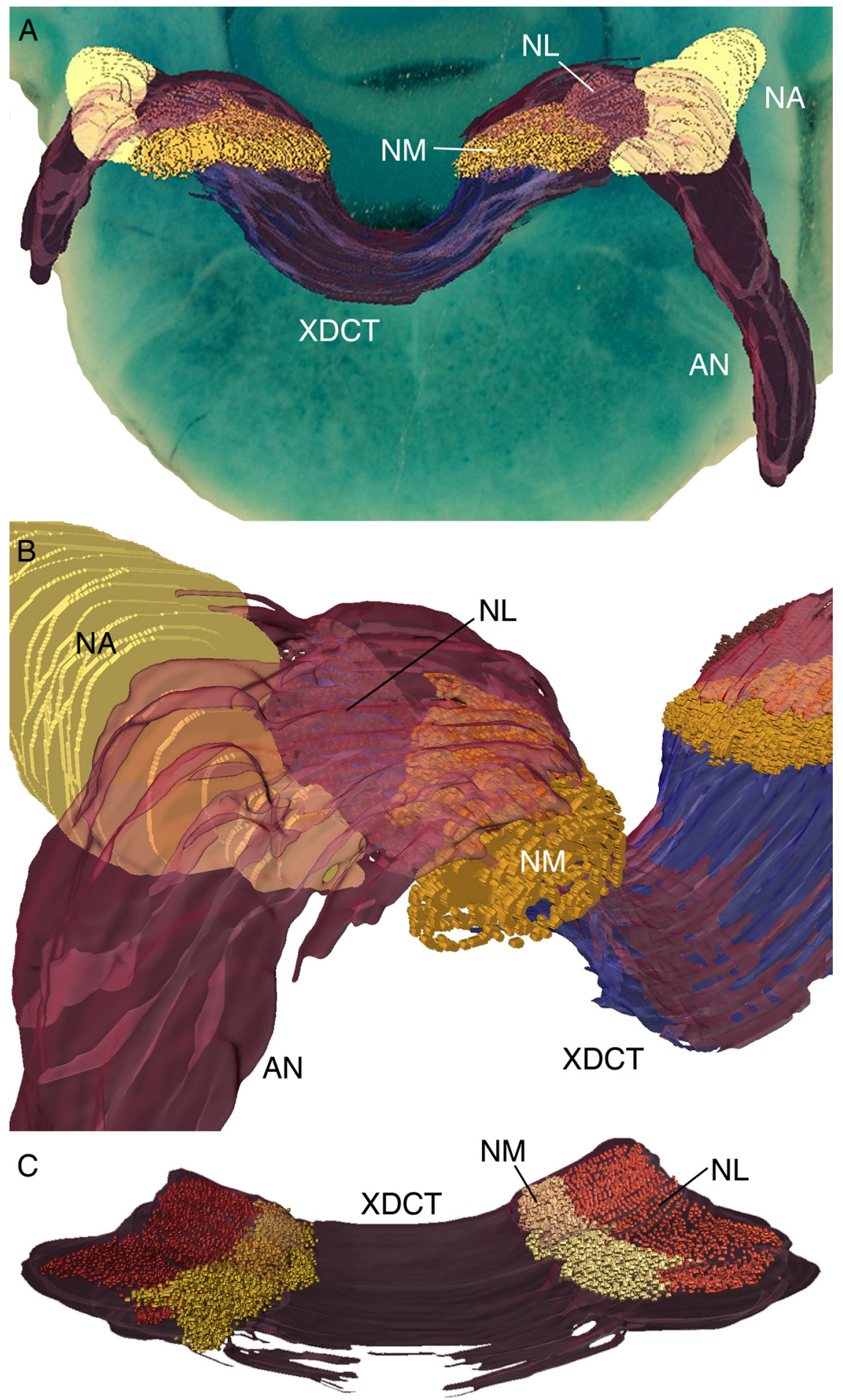

Figure 2. 3-D reconstruction of the chicken auditory brainstem nuclei. $A, 3-D$ reconstruction of $A N, N A, N M, N L$, and the XDCT in the chicken auditory brainstem depicted in front of a corresponding coronal brainstem section stained with cresyl violet. The view direction is from caudal to rostral. Note NM extends more caudally than NL. B, Close-up of the left side of the 3-D reconstruction shown in $\boldsymbol{A}$. NL lies underneath AN. AN fibers approach NM from dorsal and the contralateral projection of the XDCT continues the trajectory established by AN. C, View from caudal to rostral of both NM-NL pairs, connected by XDCT. NM: orange, NL: red, NA: yellow, fiber pathways: purple.

fresh fixative at $4^{\circ} \mathrm{C}$. Following 3 washes ( 10 min each) in $\mathrm{CB}$, brains were dissected out and $2 \mathrm{~mm}$ slabs containing NM and NL were obtained by free-hand sectioning with a razor blade. Tissue was postfixed in $1 \% \mathrm{OsO}_{4}$ in $\mathrm{CB}$ for $30 \mathrm{~min}$, followed by 3 washes in $\mathrm{CB}$, and embedded in Eponate. Semithin sections $(1-2 \mu \mathrm{m})$ were stained with $1 \%$ toluidine blue in $1 \%$ 


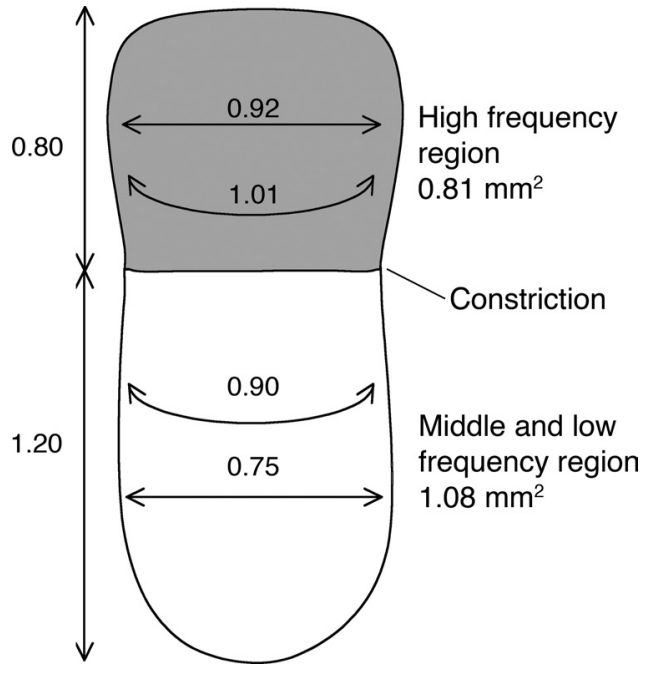

Figure 3. Dimensions of NL. The high-frequency region (rostral end, top) is separated from the middle and low-frequency regions (caudal end, bottom) by a slight constriction at a $70^{\circ}-90^{\circ}$ bend. The width of $\mathrm{NL}$ is measured straight across from edge to edge (straight arrow) and also along the curvature of NL (bent arrow). The total area of the unfolded NL of this 14-d-old animal is $1.89 \mathrm{~mm}^{2}$. In this diagram the tonotopic axis of NL is vertical and the delay line is horizontal (measurements in $\mathrm{mm}$ ).

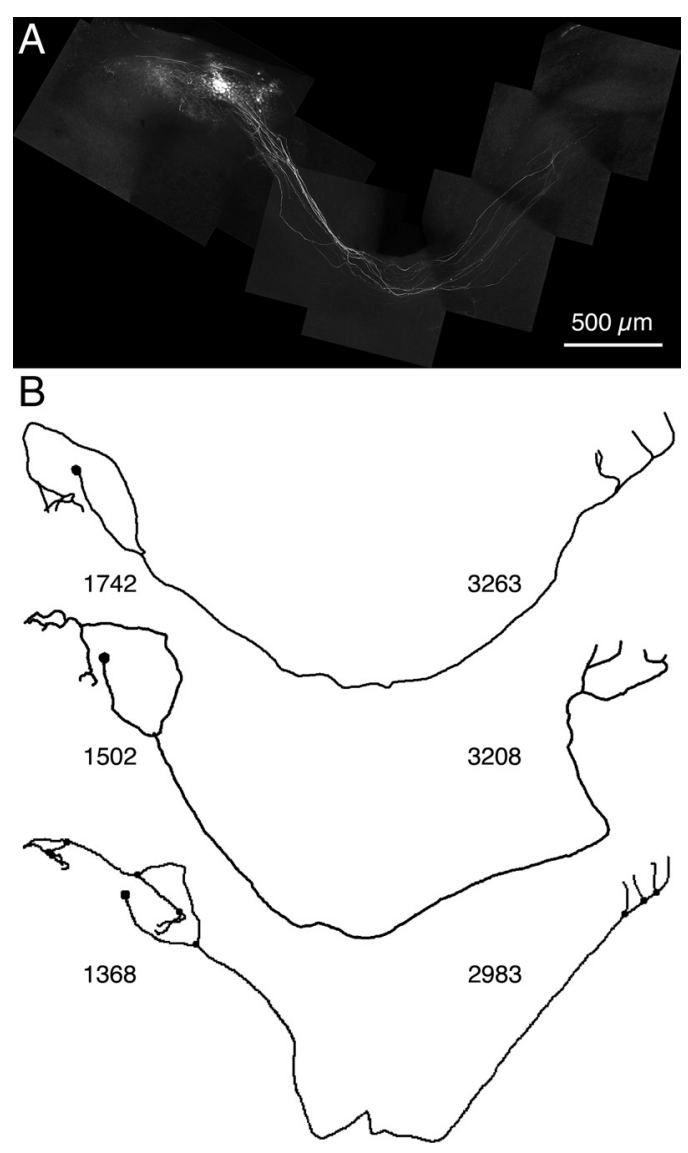

Figure 4. 3-D tracing of cells filled with fluorescent dye in acute slices reveal exact axon length at the single cell level. $A$, Serially aligned projection images of 3-D Z-stacks of confocal images in the coronal plane showing a discrete injection of Alexa Fluor 568 dextran into a cluster of cells in the left NM, as well as both the contralateral and the ipsilateral projections to bilateral NL. B, 3-D tracings of three individual cells identified. Note the cell body, the loop formed by the ipsilateral axon branch and the delay line formed by the contralateral projection. Numbers represent the distances in $\mu \mathrm{m}$ from soma to ipsilateral terminal endings (left) and from soma to the medial edge of the contralateral NL (right).
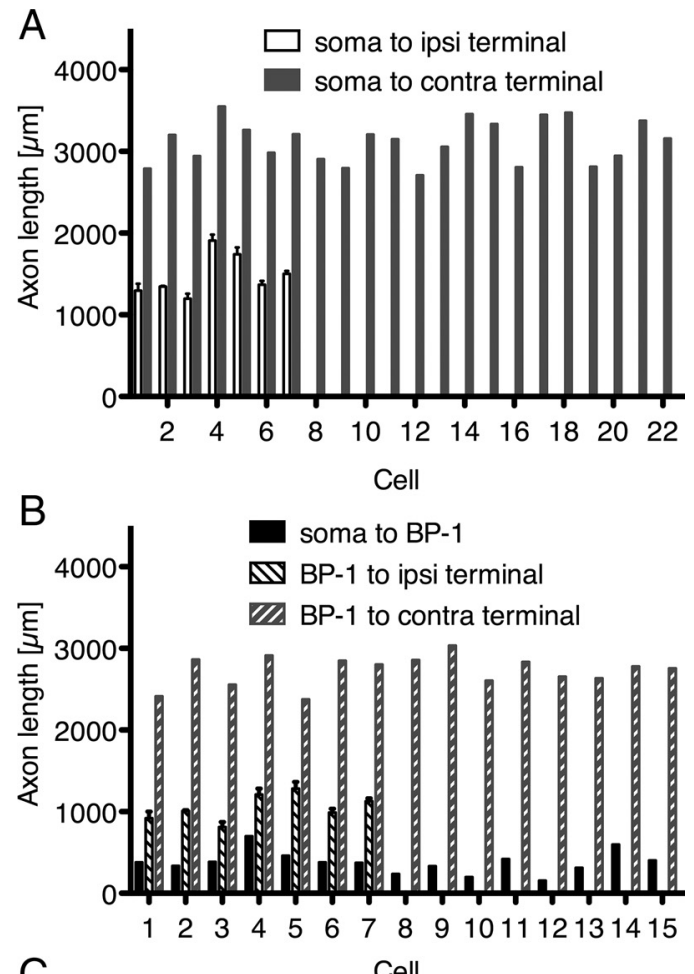

C Cell

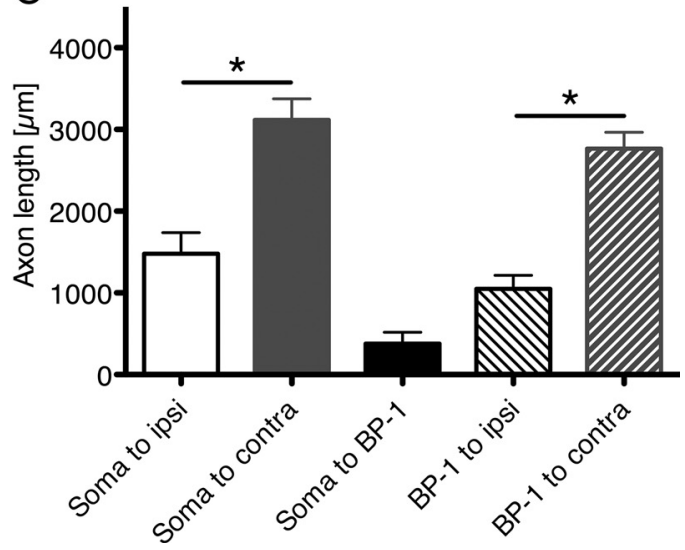

Figure 5. Lengths of axonal compartments measured in 22 single cell reconstructions. For each NM axon, the ipsilateral terminal lengths are given as an average of all the terminals. The contralateral axon was measured to the most medial terminal identified. $A$, Comparison of fiber lengths from soma to ipsilateral (white bars) and contralateral terminal (gray bars). $\boldsymbol{B}$, Axon lengths from soma to BP-1 (black bars), BP-1 to the ipsilateral terminal (white bars with black lines) and BP-1 to the contralateral terminal (gray bars with white lines). $C$, Averaged data from $A$ and $B$. On average the contralateral branch length is $1636 \mu \mathrm{m}$ longer than the ipsilateral branch. The distance from BP- 1 to the contralateral terminal is on average $1717 \mu \mathrm{m}$ longer than the segment of BP-1 to the ipsilateral terminals. Both soma to ipsilateral terminal versus soma to contralateral terminal and BP-1 to ipsilateral terminal versus BP-1 to contralateral terminal are statistically different ( $p<0.0001$, error bars show SD).

sodium borate and trimmed to area of interest before ultrathin sectioning at $90 \mathrm{~nm}$.

Ultrathin sections were mounted on 200-mesh thin bar Athene grids, stained with uranyl acetate and lead citrate and examined in a JEOL JEM 1200 EX II transmission electron microscope (JEOL). Digital images were obtained and axon diameter measured with an Olympus Morada camera (Olympus) and iTEM software (iTEM Software).

Semithin sections in the sagittal plane at the XDCT and NL were also imaged with light microscopy at $100 \times$ with a Marianas imaging system (Intelligent Imaging Innovations), incorporating a Zeiss Axiovert $200 \mathrm{M}$ microscope with an $\mathrm{X}-\mathrm{Z}$ motorized stage (ASI) and a CoolSnap HQ digital camera (Princeton Instruments). We obtained high-power im- 
ages of either NL and NM or XDCT using a $100 \times$ oil objective [numerical aperture (N.A.) 1.4] and Slidebook software with autofocus function (Intelligent Imaging Innovations). The resulting images were photomontaged in Photoshop (Adobe). The axon diameter and distance from the NL cell line were measured in ImageJ.

To avoid axon diameter measurements of fibers not cut exactly orthogonal to their long axis and to be conservative, we chose only fibers appearing round and measured diameter at the smallest value when the axon was slightly oval.

Internode distance. In 8 chickens ( $\mathrm{P} 1$ and $\mathrm{P} 3$ ) the distances between Nodes of Ranvier along individual NM axons were determined using an antibody against Caspr/paranodin/neurexin IV (NeuroMab clone K65/35, NeuroMab, University of California, Davis, Davis CA) to label paranodal proteins (Einheber et al., 1997). NM axons were labeled as described in the section above (" 3 -D tracing of individual NM axons and terminal trees"). The slices were then fixed in 4\% PFA for $30 \mathrm{~min}$ at RT. After rinsing in PBS, the slices were resectioned at 50 $\mu \mathrm{m}$ on a Vibratome (Leica VT 1000S, Leica Microsystems). After further rinsing in PBS, the sections were immunostained with 1:500 NeuroMab clone K65/35 antibody in PBS with a standard block solution [0.3\% Triton X-100 (Sigma), $5 \%$ normal goat serum] for at least $6 \mathrm{~h}$ at RT or overnight at $4^{\circ} \mathrm{C}$. The sections were then rinsed in PBS $(3 \times)$ and then exposed to the secondary antibody (1:200, Alexa Fluor 488 goat anti-mouse, Invitrogen) overnight at $4^{\circ} \mathrm{C}$ using the same block solution as with the primary antibody. After a final rinse in PBS $(3 \times)$, the sections were coverslipped with Glycergel (Dako). Sections containing both labeled NM axons and paranodal staining were imaged using a confocal microscope (Fluoview 1000, Olympus) and the resulting 3-D images loaded into Neurolucida. Nodes of Ranvier colocalizing with dye-filled axons were identified and the internode distances measured in 3-D images. If necessary, the $3-\mathrm{D}$ images were contrast-enhanced in ImageJ before the analysis in Neurolucida. The image in Figure $10 \mathrm{~B}$ was surface-rendered with Huygens Essential (Scientific Volume Imaging).

Conduction time difference calculations. To estimate actual conduction times between NM neurons and NL on each side of the brain, we used the average axon segment length data of each axon segment from our 3-D reconstruction measurements and divided them by sample conduction velocities (CV). Conduction times were then calculated as a function of each CV. Conduction time differences (NM to NL-ipsilateral vs NM to NL-contralateral) were established for XDCT conduction velocities of $2-50 \mathrm{~m} / \mathrm{s}$.

For the unadjusted conduction times, we assumed equivalent $\mathrm{CV}$ s in all NM axon segments. For the adjusted conduction times, CV was normalized to XDCT and CV in every other segment was calculated as a function of CV in XDCT multiplied with a CV correction factor. The correction factor was determined by the functions relating axon diameter and internode distance to CV (Rushton, 1951; Brill et al., 1977) of each segment relative to XDCT. All the segment conduction times for either the ipsilateral or contralateral axon length were added together and the difference in conduction times between the ipsilateral and contralateral pathway was determined.
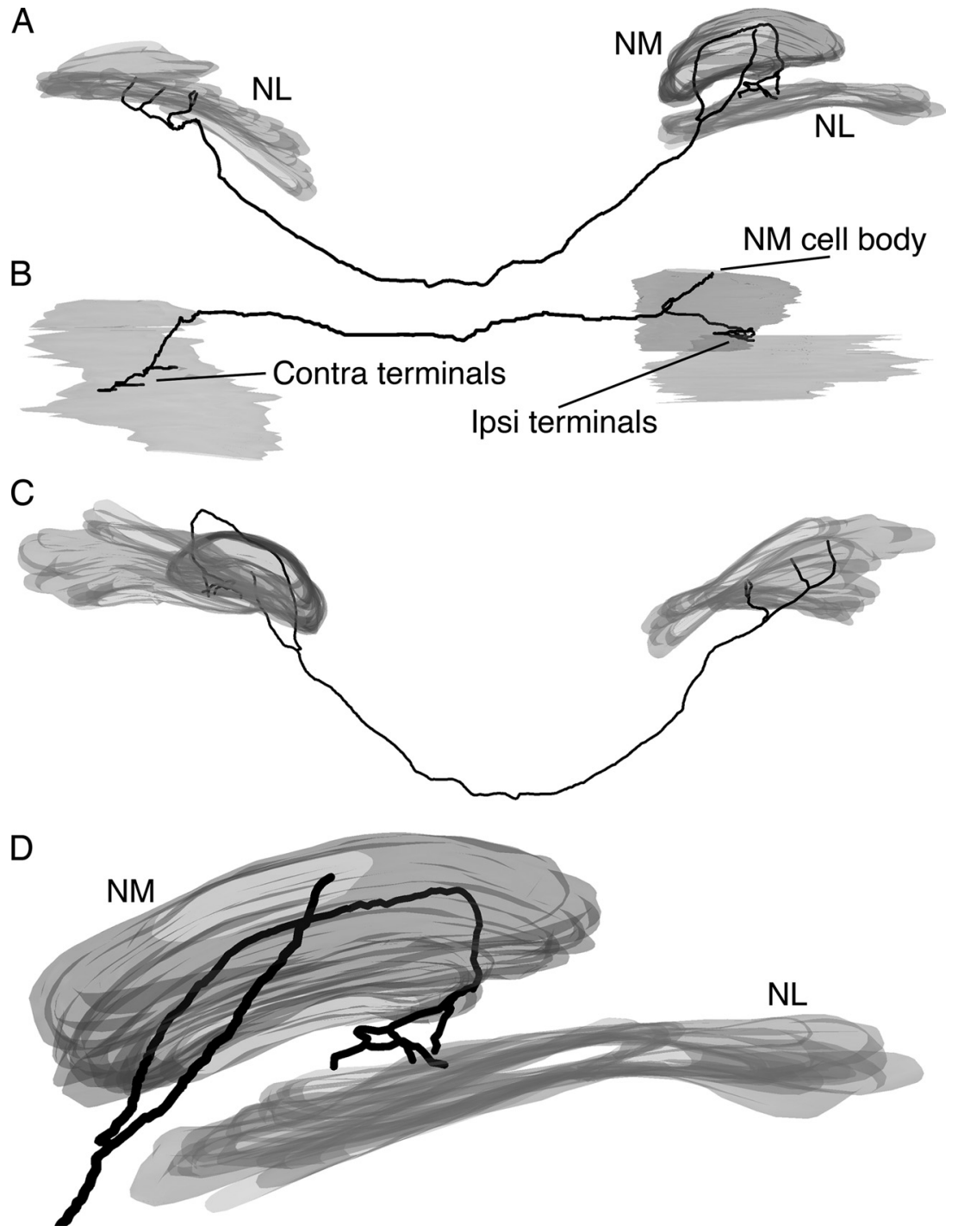

Figure 6. 3-Dreconstructions of one NM axon alongside contour outlines of the ipsilateral NM, and both the ipsilateral and contralateral A, NM axons with contours of NM, ipsilateral NL and contralateral NL viewed caudally. The ipsilateral loop and the delay line can be identified. $\boldsymbol{B}$, The ventral view of the same 3-D reconstruction shows the course of the axons along the rostrocaudal axis. Both the ipsilateral coronal plane until it reaches the contralateral NL, changes course at the medial edge of NL and continues parallel to the isofrequency endings of the ipsilateral axon branch onto the dorsal side of the ipsilateral NL.

Statistical analysis. All our statistical analyses were done with Prism 5 software (GraphPad Software). Tests for statistical significance were made by $t$ test and all error bars shown in figures represent SDs.

\section{Results}

Three major findings are presented below. First, the ipsilateral and contralateral axons from NM neurons to NL are not equal in length. This result does not support the common assumptions derived from the modified Jeffress model, that axons from the ipsi- and contralateral NM match length at the medial edge of NL. Second, measurements of axon diameter and internode distance at strategic positions along the NM axon reveal large and systematic differences in these parameters influencing conduction velocity. Third, calculations of conduction velocity (CV) using axon diameter and internode distance measurements demon- 

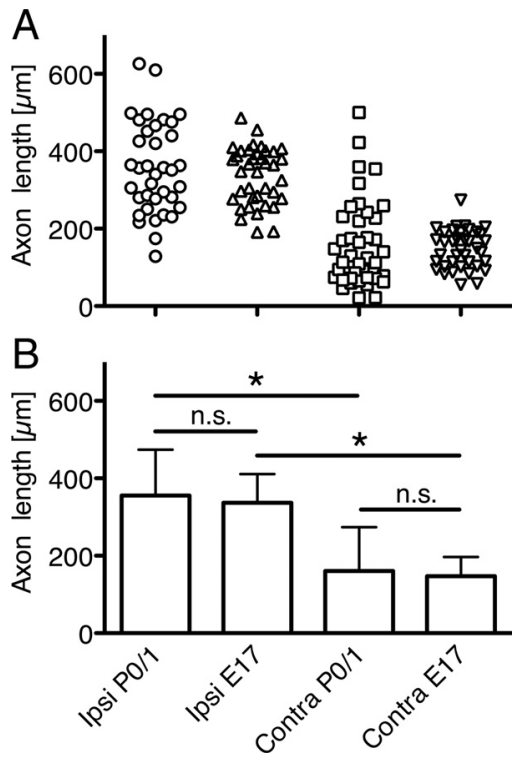

Figure 7. Comparison of NM axon terminal tree length in the dorsal and ventral neuropil of NL. A, Distribution of terminal tree axon lengths both ipsilateral and contralateral in PO/1 and E17 animals. Axon length was measured from BP-2i to the terminals for the ipsilateral tree (dorsal) and from the delay line (main axon) to the terminals for the contralateral tree (ventral). $B$, Mean values for the groups in $\boldsymbol{A}$. For the ipsilateral and contralateral tree lengths, age does not affect length, i.e., the ipsilateral and contralateral pairs are not significantly different, while for both age groups the contralateral terminal tree is significantly shorter than its ipsilateral counterpart ( $p<0.0001$, error bars show SD).

strate that the disparity in axon length can be compensated for by the parameter values that were measured.

3-D reconstruction of chicken brainstem nuclei and pathways 3-D reconstructions of NM and NL nuclei are illustrated in Figure 2 (see also movie in supplemental data, available at www. jneurosci.org). NM is situated between the concave curvature of $\mathrm{NL}$ and the floor of the IVth ventricle. The ventral aspect of NM is separated from dorsal NL by $\sim 200-300 \mu \mathrm{m}$ of neuropil. The entire NM lies slightly caudal relative to NL; the pair of nuclei is angled in the brainstem ventrocaudally to dorsorostrally (Fig. $2 C$ ) and its rostral ends are tilted medially. For most of its extent $\mathrm{NL}$ is a monolayer of bipolar cells that is curved, folded and positioned at an oblique angle. The NL resembles a saddleshaped twisted plane (Fig. $2 C$ ). There is a genu close to the rostral end of NL with a bend of $\sim 70-90^{\circ}$ that varies across animals $(n=$ 3). A 3-D reconstruction of a P7 chicken NL was measured and the dimensions of NL are illustrated on a flattened schematic in Figure 3. The whole area of one NL nucleus is $\sim 1000 \times 2000 \mu \mathrm{m}$; in the example shown the surface area is $1.89 \mathrm{~mm}^{2}$. The cell center to cell center spacing between NL cells was measured in 40 stained histological serial sections in two P15 animals. The average inter-cell distance (measured from cell center to cell center) was $27.4 \mu \mathrm{m}(\mathrm{SD} \pm 8.7 \mu \mathrm{m})$ with no apparent rostrocaudal gradient in packing density (data not shown).

\section{Integral tracing of NM neurons}

To make an integral assessment of the axonal length of single NM neurons, fluorescent markers were electroporated into small clusters of NM cell bodies in thick slices, thereby labeling their axons. Figure $4 A$ shows a montage image of multiple maximum intensity projection images, depicting a slice with an injection into the left NM. The labeled ipsilateral and contralateral branches of single cells were subsequently imaged, traced and
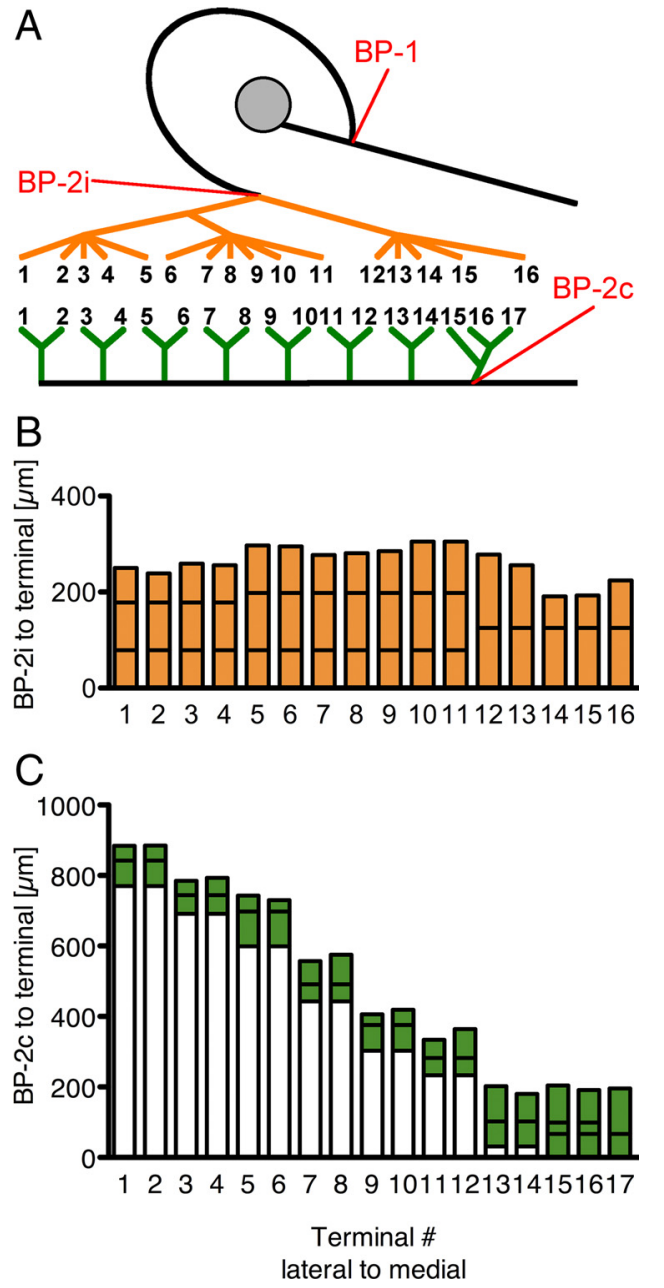

Figure 8. Quantification of terminal arborizations of NM axons onto NL at E17. A, Schematic illustrations of ipsilateral (top, orange) and contralateral (bottom, green) arborizations of an NM neuron onto NL (E17) (Young and Rubel, 1983). Indicated are BP-1, BP-2i, and BP-2C (see Fig. $1 B$ ) and numbered terminal clusters. $\boldsymbol{B}$, Graph of the measured arborization distances from the second branch point on the ipsilateral side (BP-2i) to the terminal endings (orange). Black horizontal lines within the columns indicate branch points. All ipsilateral projections are of similar length (mean: $262 \pm 36 \mu \mathrm{m}$ ). C, Graph of measured arborizations from BP-2c to the terminal endings. White bar segments show the increasing length of the main axon medial to lateral, forming the delay line. Shown in green are the axon segments branching off the main axon (mean: $136 \pm 35 \mu \mathrm{m}$ ).

measured. Three representative tracing examples are shown in Figure $4 B$. For each tracing, the cell body, the ipsilateral loop and terminals, as well as the contralateral axon and terminals can be identified. The numbers indicate the axon branch length (in $\mu \mathrm{m}$ ) from the soma to the respective ipsilateral and contralateral terminal. In all cases the contralateral distance was measured to its most medial contralateral NL neuronal termination.

The main result of axon tracing measurements is presented in Figure 5A. Data are derived from 22 cells where axon length was determined from the NM soma to the contralateral NL. In 7 of these cells the length of the axon branch from the soma to ipsilateral axon endings was measured. Again, data for the contralateral axon is given as the distance to the most medial terminal identified and the length of the ipsilateral axon is given as the average across all terminals identified.

The distance from the soma to the point where the axons first bifurcates was determined as well (BP-1) (see Materials and Methods and Fig. $1 B$ ) and the distance from BP-1 to the most 
medial terminal identified in 15 cells (Fig. $5 B)$. In 7 of those cells we additionally measured the axon segment length from BP-1 to the ipsilateral terminal (Fig. 5B).

The average length $( \pm S D)$ for the ipsilateral NM axonal projection was $1480 \pm 257 \mu \mathrm{m}$ and the mean of the contralateral projection was $3116 \pm 258 \mu \mathrm{m}$ (Fig. $5 C$ ). Thus, the shortest (most medial) contralateral NM-NL projection averages $>1600 \mu \mathrm{m}$ longer than the corresponding ipsilateral projection. The distance from the soma to BP-1 has a mean of $378 \pm 139 \mu \mathrm{m}$. Consequently, the mean value for the distance from BP-1 to the ipsilateral terminal is $1049 \pm 166$ $\mu \mathrm{m}$ and $2766 \pm 200 \mu \mathrm{m}$ to the most medial contralateral terminal (Fig. 5C). There is a profound and statistically significant length difference between the axon branch extending ipsilaterally and the branch extending contralaterally (Fig. $5 C, p<0.0001)$.

The 3-D reconstructions also showed that NM axons project overall slightly rostrally (Fig. 6). The ipsilateral loop originates in an NM neuron that is located more caudally in the brainstem than its terminal field, consistent to what the tonotopic organization of both nuclei would dictate (Rubel and Parks, 1975). The contralateral branch projects across the midline in the coronal plane. When it reaches the medial edge of NL it angles rostrally, to travel along an isofrequency contour of NL, confirming previous findings by Young and Rubel (1983).

\section{Analysis of terminal trees}

Axon terminal trees from 3-D reconstructions of NM axons in P0/1 animals were measured and compared with archival material published previously (Young and Rubel, 1983, 1986). Figure $7 A$ shows the distribution of axon length in the ipsilateral terminal tree (BP-2i to terminal, mean $356 \pm 118 \mu \mathrm{m}, n=37$ ) and of axon length in the contralateral terminal tree, measured from the main axon branch coursing ventral to NL to the terminal (mean $168 \pm 113 \mu \mathrm{m}, n=40$ ) (Fig. $7 B$ ). The same analysis was obtained from the archival E17 data (Fig. $7 A$ ). Here the mean for the ipsilateral terminal tree is $337 \mu \mathrm{m}( \pm 74 \mu \mathrm{m}, n=38)$ and for the contralateral terminal tree $147 \mu \mathrm{m}( \pm 49 \mu \mathrm{m}, n=38)$ (Fig. $7 B$ ). Therefore, for both age groups the ipsilateral terminal trees are almost $200 \mu \mathrm{m}$ longer than the contralateral terminal trees $(p<0.0001)$. There is no significant difference of terminal tree length between the two age groups (ipsilateral: $p=0.4142$, contralateral: $p=0.4815$ ).

The example shown in Figure $8 \mathrm{~A}$ depicts a graphical schematic of the ipsilateral and contralateral terminal trees of an E17 animal in detail. Distances were measured from soma to BP-1, from BP-1 to BP-2i, from BP-2i to the ipsilateral terminals (orange) and from BP-2c to the contralateral terminals (white: delay line, main axon; green: contralateral terminal tree). The distance from soma to BP-1 was $354 \mu \mathrm{m}$. The distances from BP-2i to the they approach NL.
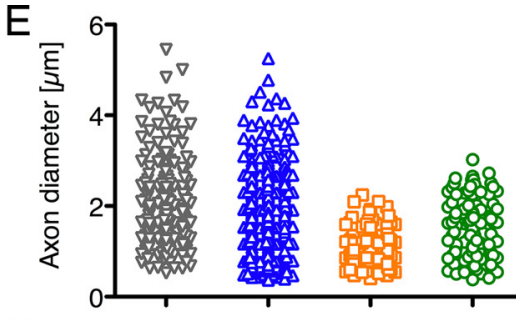

\section{$F$}
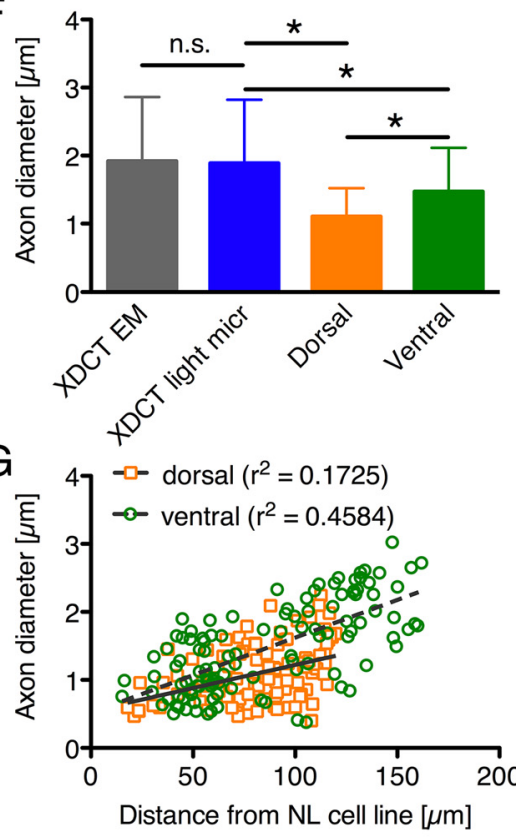

Figure 9. Axon diameter in the XDCT, and the dorsal and ventral terminal fields. $\boldsymbol{A}$, Schematic of the chicken auditory brainstem indicating the location where the NM axon diameter was determined. Distributions of axon diameter were measured in the XDC XDCT, and dorsal axon diameter was smaller than in the ventral terminal tree. ( $p<0.0001$, error bars indicate SD). G, Axon iameter in the terminal trees in relation to distance from the $\mathrm{NL}$ cell line. Diameters of both dorsal and ventral axons decrease as

terminal endings are plotted in Figure $8 B$ and average $262 \mu \mathrm{m}$ ( \pm 36 ), and are very similar across the nucleus from the most medial to the most lateral NL neuron. The complete lengths of the ipsilateral pathways from BP-1 to the ipsilateral terminals are $848 \mu \mathrm{m}$ on average. The ventral terminal arborization line (delay line), i.e., from BP-2c to the most lateral terminal, measures 885 $\mu \mathrm{m}$ (Fig. $8 \mathrm{C}$ ). The projections from branching points along the delay line running parallel to the NL ventrally to the terminal endings average $136 \mu \mathrm{m}$ ( \pm 35 ) (Fig. 8C, green segments). As expected (Young and Rubel, 1986) the distance from BP-2c to terminal endings on the ventral side increases from medial to lateral.

\section{Axon diameter}

Since axon diameter influences conduction velocity and differences in diameter could compensate for differences in the NM axon path lengths to the ipsilateral and contralateral NL, NM axon diameter was measured in sagittal semithin and ultrathin sections in different segments of the NM axon (Fig. 9A). NM axon diameter was evaluated in the XDCT near the midline (Fig. $9 B$ ), within the ipsilateral terminal tree branching dorsal onto NL 

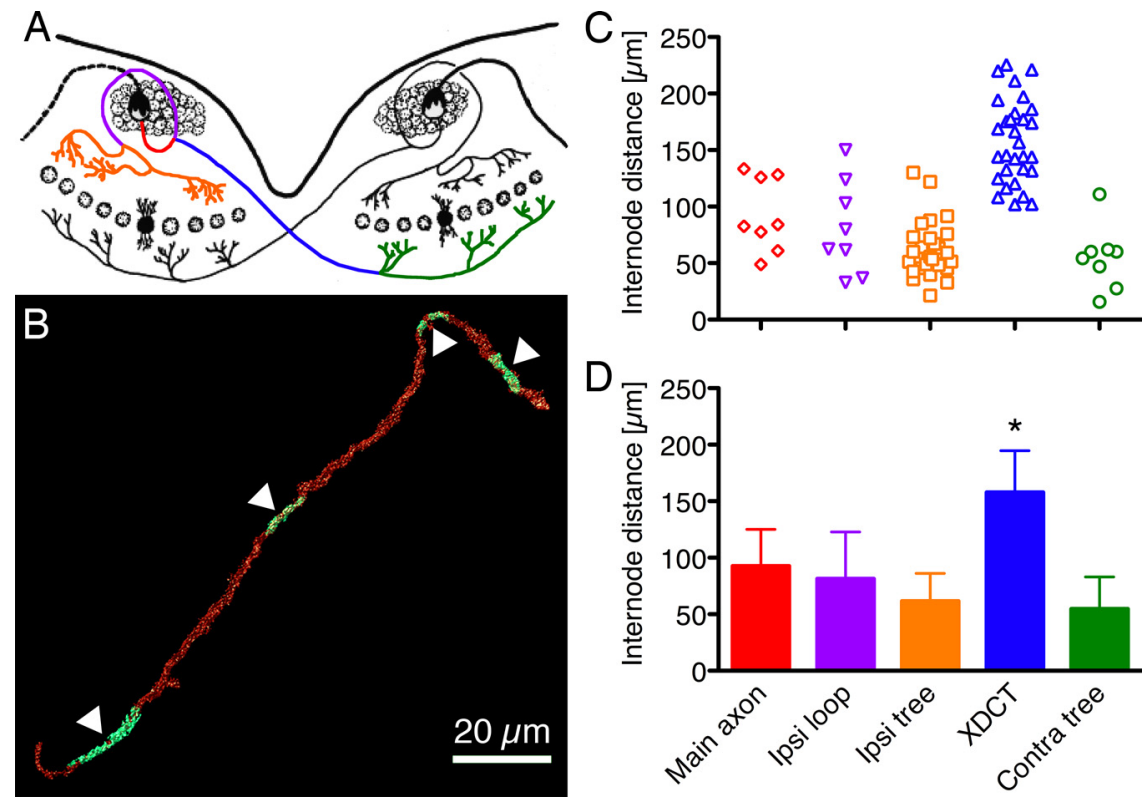

Figure 10. Internode distances in the NM axon. $\boldsymbol{A}$, Schematic graphic of the chicken auditory brainstem indicating the different axon segments of NM where internode distances were measured (red: proximal segment, purple: ipsilateral loop, orange: ipsilateral tree, blue: XDCT, green: contralateral tree). $\boldsymbol{B}$, Surface rendered image of an axon segment in the ipsilateral loop filled with fluorescent dye (red) and counterstained with an antibody labeling a paranodal protein (green). Nodes of Ranvier can be identified (white arrowheads). C, Distribution of measured internode distances in the different axon segments. D, Mean values for the data in C show that internode distance in the XDCT is significantly larger than in every other group ( $p<0.0001$, error bars indicate SD).

(Fig. 9C), and within the contralateral terminal tree branching ventral onto NL (Fig. 9D). Axon diameter measurements in the NL neuropil were also made as a function of distance from the NL cell line (Fig. 9G). Experiments in which we electroporated dye into the XDCT confirmed the homogenous population of axons, as only fibers extending to NM and NL were labeled (data not shown). We refrained from measuring axon diameter in the ipsilateral loop, i.e., in the segment from BP-1 to BP-2i. The mixed content of fibers in this area, containing auditory nerve axons as well as axons to the lateral lemniscal nuclei and midbrain, would make it impossible to get a clean database of NM to NL axons for this segment.

Three of the data groups shown in Figure 9 are from measurements made from semithin, osmium fixed, plastic embedded sections at maximum light microscopic resolution $[100 \times$ (N.A. 1.4) objective]. To validate our measurement method using light microscopy, we measured axon diameter in electron microscopic images and light microscopic images at the midline (in the XDCT) from the same tissue (Fig. 9E, F; see Materials and Methods). We found no significant difference between the two groups $(p=0.7280)$ with similar values of means and SDs (EM: $1.917 \pm 0.943 \mu \mathrm{m}$; light microscopy: $1.889 \pm 0.933 \mu \mathrm{m})$.

Figure 9, $E$ and $F$, presents the results of measurements of axon diameters in the XDCD and the terminal arbors approaching the dorsal and ventral neuropil regions of NL. Average axon diameters dorsal $(1.104 \pm 0.417 \mu \mathrm{m})$ and ventral of NL (1.470 \pm $0.646 \mu \mathrm{m})$ are both significantly smaller than the mean axon diameter along the midline $(p<0.001)$. Dorsal axon diameters are also significantly smaller than diameters in ventral terminal tree $(p<0.001)$.

Axon diameter measurements in the NL neuropil as a function of their distance from the NL cell line (Fig. 9G) show a decrease in diameter as they approach NL (Fig. 9G). The two distribution correlations are significant $(p<0.001)$ and the re- gression line slopes are significantly different from each other $(p<0.05)$. However, if similar distance ranges $(\leq 120$ $\mu \mathrm{m}$ distance from NL cell line) are considered, i.e., datapoints of the ventral group with $>120 \mu \mathrm{m}$ distance from the NL cell line are excluded, the regression line slopes are not significantly different from each other $(p=0.19)$, while the distributions are $(p<0.05)$. The $r^{2}$ value for the linear regression of ventral axon diameters within $120 \mu \mathrm{m}$ from the NL cell line is 0.2491 .

\section{Internode distances}

Another important factor regulating conduction velocity of axons is the distance between the nodes of Ranvier; the farther the distance between nodes, the faster the conduction velocity (Brill et al., 1977). Nodes in the NM axon were evaluated by filling axons with a fluorescent dye and counterstaining the resectioned tissue containing labeled axons with an antibody against a paranodal protein. Distances between the nodes were measured in 3-D using confocal microscopy along 5 different segments of the NM axon (Fig. $10 \mathrm{~A}$ ). Figure $10 \mathrm{~B}$ shows an axon segment of the ipsilateral loop (axon: red, paranodal protein: green, white arrowheads indicate Nodes of Ranvier).

The measurements shown in Figure 10, $C$ and $D$, provide the distributions of internode distance $(C)$ and the means with SDs (D) (proximal segment $92.69 \pm 32.42$, ipsi loop $81.29 \pm 41.54$, ipsi terminal tree $61.59 \pm 24.58$, XDCT $157.8 \pm 36.83$, contra terminal tree $54.69 \pm 28.29$, all $\mu \mathrm{m}$ ). These data reveal that the internode distances along the XDCT are significantly larger than all other segments $(p<0.0001)$, again indicating the fastest conduction velocity in the XDCT.

\section{Conduction time difference}

Next, we evaluated the data presented above for the difference in conduction time between the ipsilateral and contralateral axon. To accomplish this, axon diameter and internode distance were normalized to the values found in the XDCT and the adjusted conduction times calculated for all segments along the NM axon (see Material and Methods).

Figure $11 A-D$ shows the calculated conduction times for each axon segment as a function of conduction velocities along XDCT. The conduction velocity for the contralateral axon along the ventral side of NL was measured in a chicken brainstem slice preparation by Overholt et al. (1992). Based on these data we assume that the conduction velocity will be between $3.0-8.8 \mathrm{~m} / \mathrm{s}$.

Figure 11, $A$ and $B$, shows conduction times for an axon with uniform conduction velocity. Each axon segment contributes to the overall conduction time purely by its length. Figure 11, $C$ and $D$, shows conduction times for an axon in which conduction velocities vary and are adjusted depending on axon diameter and internode distance. The graphs in Figure 11, $A$ and $C$, show values for the ipsilateral branch and Figure $11, B$ and $D$, show values for the contralateral branch. Figure $11 E$ is derived from this analysis and shows the difference in arrival time of action potentials between the ipsilateral and contralateral NL based on whether ve- 
A

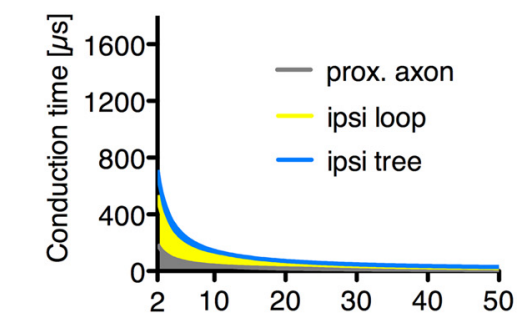

C

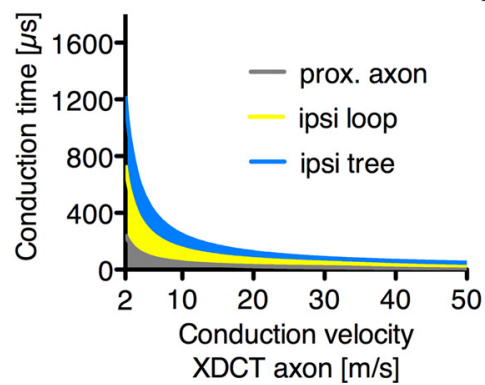

B

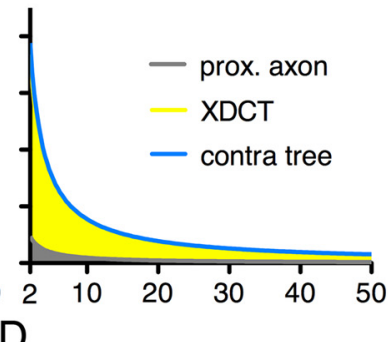

D

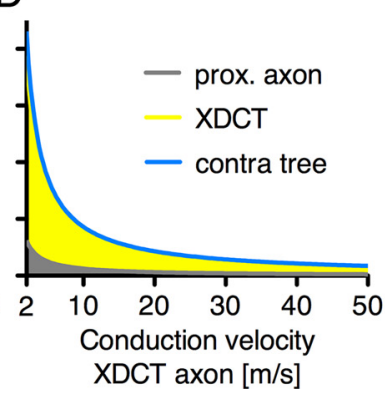

$\mathrm{E}$

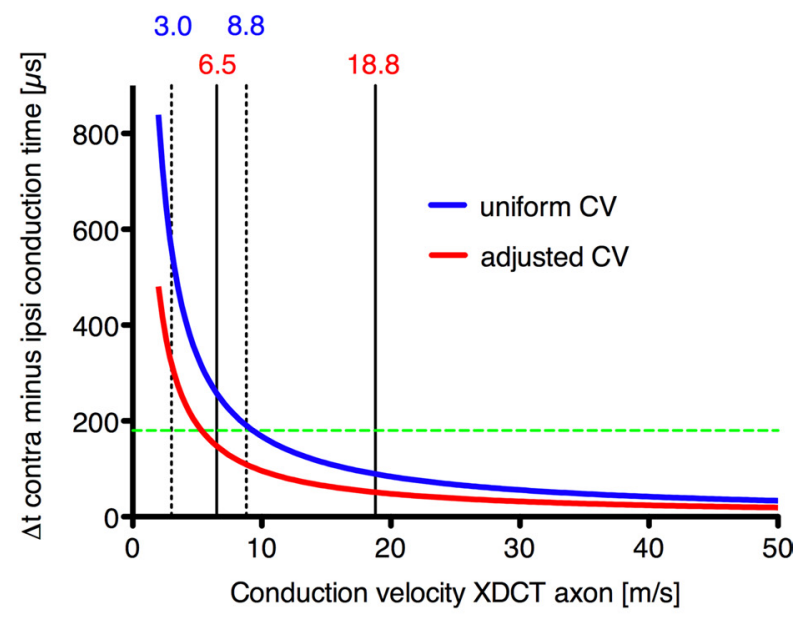

Figure 11. Conduction times and conduction time difference. $\boldsymbol{A}, \boldsymbol{B}$, Conduction times for axon segments of the ipsi- and contralateral NM axon when conduction velocity (CV) is assumed to be equal throughout the entire axon. Conduction time is given as a function of conduction velocity in XDCT. C, D, Conduction times for axon segments in an NM axon in which conduction velocities vary between different axon segments depending on measured axon diameter and internode distance. We calculated conduction times as a function of conduction velocity in the XDCT part of the axon with values ranging from 2 to $50 \mathrm{~m} / \mathrm{s}$. Conduction velocities in the other axon segments were adjusted according to axon diameter and internode distance (see Materials and Methods). Conduction time in $C, D$ is increased compared to $A, D$, most prominently in the ipsilateral terminal tree. $E$, Conduction time difference between the ipsilateral and contralateral axon as a function of conduction velocity in XDCT, calculated for NM axons with uniform conduction velocity (blue curve) and for axons in which different segments have different conduction velocities (solid red curve, see Materials and Methods). For uniform conduction velocity values between 3.0 and $8.8 \mathrm{~m} / \mathrm{s}$ (dashed vertical lines, Overholt et al., 1992), the calculated difference in conduction time is entirely outside the range of physiological ITDs ( $\pm 180 \mu \mathrm{s}$, dashed green horizontal line). Considering adjusted conduction velocities $(\boldsymbol{C}, \boldsymbol{D})$, the difference becomes smaller (solid red curve). Taken into account that the conduction velocities in the XDCT and along the NL neuropil differ, the conduction time difference falls within the physiological range (for $3.0-8.8 \mathrm{~m} / \mathrm{s}$ along NL the adjusted conduction velocity in XDCT is $6.5-18.8 \mathrm{~m} / \mathrm{s}$, solid vertical lines).

locities are assumed to be equivalent in all axon segments of the NM axonal trajectory (blue line, unadjusted, data from Fig. $11 A, B)$ or velocities are compensated by differences in axon diameter and internodal distances (red line, adjusted, data from Fig. $11 C, D)$. The dashed horizontal green line shows the maximum range of physiological ITDs in chicks at $\pm 180 \mu$ s, defined by the speed of sound, the inter ear distance and the interaural canal frequency-dependent effects (Calford and Piddington, 1988; Overholt et al., 1992; Hyson et al., 1994; Hyson, 2005).

Assuming equal conduction velocity throughout the entire length of the NM to NL axon branches, the difference between ipsilateral and contralateral conduction time is too large to be within the physiological relevant range. For conduction velocity values between 3.0 and $8.8 \mathrm{~ms}$ (Overholt et al., 1992) (Fig. 11, dashed vertical lines), the calculated difference in conduction time is entirely outside the physiological range of ITDs. With the adjusted conduction times from Figure 11, $C$ and $D$, the difference in conduction time between ipsilateral and contralateral is substantially decreased. Here, the conduction velocities in the XDCT and along the NL neuropil differ. Therefore, based on measured conduction velocities in the NL neuropil (Overholt et al., 1992), as well as the differences in axon diameter and internode distance, it is assumed that the axons in the XDCT have a conduction velocity between 6.5 and $18.8 \mathrm{~m} / \mathrm{s}$ (mean $11.9 \mathrm{~m} / \mathrm{s}$, solid vertical lines). Given these values, the difference between ipsilateral and contralateral conduction time falls into the physiological range (Fig. $11 E$ ). Hence, the variations in axon diameter and internode distance can provide a means to compensate for the axon length disparity.

\section{Discussion}

In this study we measured axon length in the chicken brainstem sound localization circuit responsible for the processing of ITDs, as well as parameters responsible for conduction velocity, specifically axon diameter and internode distance. Surprisingly, axon length dimensions do not to comply with the proposed modified Jeffress model (Overholt et al., 1992), as the contralateral axon is $>1600 \mu \mathrm{m}$ longer than the ipsilateral one, making coincidence detection based on travel distance impossible. However, the data indicate that variations in both axon diameter and internode distances may counterbalance the offset in axon length. This axon length disparity and anatomical evidence for temporal compensation provokes a reassessment of current thinking about the organization of the avian and mammalian sound localization circuits responsible for ITD coding.

In accord with the hitherto proposed model embodied by the NM-NL circuit (Overholt et al., 1992), our results illustrate similar lengths of axons from NM onto the dorsal NL dendrites and confirm that each of the contralateral axons forms successive series of short collaterals stemming from a parent axon coursing immediately ventral to NL neurons orthogonal to the tonotopic axis. These collaterals form a "delay line" of axon terminals on NL neurons from the contralateral NM. The most interesting and unforeseen observation of this study is a large and reliable difference in the lengths of contralateral and ipsilateral pathways from NM to NL, which does not comply with the proposed modified Jeffress model. In their seminal paper about the barn owl sound localization circuit, Carr and Konishi (1990) also noted a difference in axon lengths from NM to the ipsilateral and contralateral 
NL. The differences ( $\sim 1 \mathrm{~mm}$, and a much larger head) were not as great as those reported here, but that may be due to the fact that three-dimensional reconstructions were not attempted. In this paper, Carr and Konishi (1990) also foreshadowed our findings of variations in axon caliber and internodal differences, noting that these two parameters varied along the pathways and may modulate conduction velocities in meaningful ways. Physiological data demonstrate the existence of neurons responding best to 0 ITD (Köppl and Carr, 2008). Indeed, rough calculations of the conduction velocity for the ipsilateral and contralateral axon using response delay data from Köppl and Carr (2008), their Figure 4 and from Köppl (1997), her Figure 10 yield values that average at 3.8 and $8 \mathrm{~m} / \mathrm{s}$, respectively. Not only are the resulting values for the contralateral axon twice as large, the conduction velocity for the contralateral axon is also consistent with the values proposed for the XDCT in this paper (Fig. 11E). Given the difference in length between ipsi- and contralateral axon collaterals, the encoding of ITDs in the physiological range for the chicken is impossible unless other morphological or physiological features regulate the timing in these circuits. In myelinated axons, their diameter is linearly proportional to conduction velocity (Gasser and Grundfest, 1939; Rushton, 1951; Hutchinson et al., 1970). Our data show significantly smaller axon diameters in the terminal trees compared to the major branches of the axons as they traverse the XDCT. Additionally, the ipsilateral terminal tree has an average axon diameter that is only three quarters of the average diameter in the ventral terminal tree, further delaying the arrival of ipsilateral action potentials at NL relative to the contralateral signal. This effect is amplified by the fact that the average axon length in the ipsilateral terminal tree is more than double the length of the mean axon in the ventral terminal tree. We did not measure axon diameter in the ipsilateral loop, but variations in this segment might enable the system to further alter conduction velocities and to slow down the signal on the ipsilateral side.

Brill and colleagues showed that conduction velocity increases linearly with internode distance to up to $2000 \mu \mathrm{m}$ (Brill et al., 1977). Moreover, when the ratio of internode distance (L) and axon diameter $(\mathrm{d})$ is small $(<150)$, conduction velocity is quite sensitive to variations in L/d (Brill et al., 1977). Carr and Konishi (1990) compared internode distance of axons as they traverse the cell body layers within the owl NL with axons outside NL, and also report shorter internode distances of the axons within NL. At that time, methods to label individual axons were not readily available; hence the origin of the axons they measured could not be confirmed. We were able to measure internode distances in different segments of labeled NM axons and found significant variations along the NM axon. In particular, internode distances along XDCT is significantly larger compared to other parts of the NM axon by at least 50\%. It also appears that internode distances within the terminal arbors on both the ipsilateral/dorsal axons and the contralateral/ventral axons are smaller than the main branches leading up to these terminal branches. Again, this disparity of terminal tree length enhances the effect of short internode distances along the terminal endings and contributes to delaying the propagating signal ipsilateral relative to the contralateral side of the brain. Taking into account the axon diameter variations and the resulting sensitivity to $\mathrm{L} / \mathrm{d}$ ratio, there seems to be ample possibility for variations in signal speed.

In summary, our results show that at least two parameters influencing conduction velocity, axon diameter and internode distances, are regulated at different sites within individual axons of NM neurons to specifically adjust the conduction velocities of the ipsilateral and contralateral signals, presumably to optimize coincidence detection. Slowing down the propagating signal in the ipsilateral part of the axons allows the contralateral signal to "catch up" and enables encoding of ITDs in the physiological range in NL neurons. The regulation of these axonal parameters within individual axons seems quite remarkable from a cell biological point of view, but it is not unprecedented. Measurements in other neuronal systems have been shown to provide temporal compensation for conduction distance disparities. For example, in the electromotor system in fish internode distance is adjusted so that conduction velocity compensates for different axon lengths (Bennett, 1970). In rat Purkinje cells, conduction velocity is varied to enable isochronicity of different length climbing fibers (Sugihara et al., 1993). Other parameters, such as myelin sheet thickness (Rushton, 1951), may also influence signal propagation in the NM-NL circuit. Moreover, one might inquire if there is a period of adjustment for one or several of these morphological features during development, wherein these circuits are temporally sharpened and compensated for changes in head size to optimize discrimination of binaural timing differences.

We show that axon length alone cannot be solely responsible to compensate for external ITDs in the chicken sound localization circuit. This unexpected result has implications for the mammalian brainstem binaural system as well. It is noteworthy that the mechanism responsible for low-frequency sound localization in mammals has recently come under dispute. There is only marginal anatomical evidence for delay lines in mammals (Smith et al., 1993; Beckius et al., 1999) and a proposed alternative mechanism, incorporating the glycinergic inputs to MSO, requires a very fast inhibitory input $\left(\tau_{\text {decay }}=0.1 \mathrm{~ms}\right.$ ) (Brand et al., 2002) that has not been confirmed by physiological measurements (Magnusson et al., 2005). Variations of parameters such as axon diameter, internode distance, and others (Pecka et al., 2008) in the mammalian brainstem might be responsible for precise adjustments of physiological delays, thereby creating the framework and adjustments of the ITD detection circuit.

\section{References}

Beckius GE, Batra R, Oliver DL (1999) Axons from anteroventral cochlear nucleus that terminate in medial superior olive of cat: observations related to delay lines. J Neurosci 19:3146-3161.

Bennett MV (1970) Comparative physiology: electric organs. Annu Rev Physiol 32:471-528.

Brand A, Behrend O, Marquardt T, McAlpine D, Grothe B (2002) Precise inhibition is essential for microsecond interaural time difference coding. Nature 417:543-547.

Brill MH, Waxman SG, Moore JW, Joyner RW (1977) Conduction velocity and spike configuration in myelinated fibres: computed dependence on internode distance. J Neurol Neurosurg Psychiatry 40:769-774.

Burger RM, Cramer KS, Pfeiffer JD, Rubel EW (2005) Avian superior olivary nucleus provides divergent inhibitory input to parallel auditory pathways. J Comp Neurol 481:6-18.

Calford M, Piddington R (1988) Avian interaural canal enhances interaural delay. J Comp Physiol A Neuroethol Sens Neural Behav Physiol 162:503-510.

Carr CE, Konishi M (1990) A circuit for detection of interaural time differences in the brain stem of the barn owl. J Neurosci 10:3227-3246.

Einheber S, Zanazzi G, Ching W, Scherer S, Milner TA, Peles E, Salzer JL (1997) The axonal membrane protein Caspr, a homologue of neurexin IV, is a component of the septate-like paranodal junctions that assemble during myelination. J Cell Biol 139:1495-1506.

Gasser H, Grundfest H (1939) Axon diameters in relation to the spike dimensions and the conduction velocity in mammalian A fibres. Am J Physiol 127:393-414

Haas K, Sin WC, Javaherian A, Li Z, Cline HT (2001) Single-cell electroporation for gene transfer in vivo. Neuron 29:583-591.

Harris DM, Rotche R, Freedom T (1990) Postnatal growth of cochlear spiral in Mongolian gerbil. Hear Res 50:1-6. 
Harris DM, Seidl AH, Rubel EW (2008) The shape of laminaris. Assoc Res Otolaryngol. Abs.: 1166.

Hutchinson NA, Koles ZJ, Smith RS (1970) Conduction velocity in myelinated nerve fibres of Xenopus laevis. J Physiol 208:279-289.

Hyson RL (2005) The analysis of interaural time differences in the chick brain stem. Physiol Behav 86:297-305.

Hyson RL, Overholt EM, Lippe WR (1994) Cochlear microphonic measurements of interaural time differences in the chick. Hear Res 81:109-118.

Jeffress LA (1948) A place theory of sound localization. J Comp Physiol Psychol 41:35-39.

Jhaveri S, Morest DK (1982) Neuronal architecture in nucleus magnocellularis of the chicken auditory system with observations on nucleus laminaris: a light and electron microscope study. Neuroscience 7:809-836.

Joseph AW, Hyson RL (1993) Coincidence detection by binaural neurons in the chick brain stem. J Neurophysiol 69:1197-1211.

Köppl C (1997) Phase locking to high frequencies in the auditory nerve and cochlear nucleus magnocellularis of the barn owl, Tyto alba. J Neurosci 17:3312-3321.

Köppl C, Carr CE (2008) Maps of interaural time difference in the chicken's brainstem nucleus laminaris. Biological cybernetics 98:541.

Kuba H, Koyano K, Ohmori H (2002) Synaptic depression improves coincidence detection in the nucleus laminaris in brainstem slices of the chick embryo. Eur J Neurosci 15:984-990.

MacDonald GH, Rubel EW (2008) Three-dimensional imaging of the intact mouse cochlea by fluorescent laser scanning confocal microscopy. Hear Res 243:1-10.

Magnusson AK, Kapfer C, Grothe B, Koch U (2005) Maturation of glycinergic inhibition in the gerbil medial superior olive after hearing onset. J Physiol 568:497-512.
McAlpine D, Jiang D, Palmer AR (2001) A neural code for low-frequency sound localization in mammals. Nat Neurosci 4:396-401.

Overholt EM, Rubel EW, Hyson RL (1992) A circuit for coding interaural time differences in the chick brainstem. J Neurosci 12:1698-1708.

Pecka M, Brand A, Behrend O, Grothe B (2008) Interaural time difference processing in the mammalian medial superior olive: the role of glycinergic inhibition. J Neurosci 28:6914-6925.

Reyes AD, Rubel EW, Spain WJ (1996) In vitro analysis of optimal stimuli for phase-locking and time-delayed modulation of firing in avian nucleus laminaris neurons. J Neurosci 16:993-1007.

Rubel EW, Parks TN (1975) Organization and development of brain stem auditory nuclei of the chicken: tonotopic organization of n. magnocellularis and n. laminaris. J Comp Neurol 164:411-433.

Rushton WA (1951) A theory of the effects of fibre size in medullated nerve. J Physiol 115:101-122.

Smith PH, Joris PX, Yin TC (1993) Projections of physiologically characterized spherical bushy cell axons from the cochlear nucleus of the cat: evidence for delay lines to the medial superior olive. J Comp Neurol 331:245-260.

Sorensen SA, Rubel EW (2006) The level and integrity of synaptic input regulates dendrite structure. J Neurosci 26:1539-1550.

Sugihara I, Lang EJ, Llinás R (1993) Uniform olivocerebellar conduction time underlies Purkinje cell complex spike synchronicity in the rat cerebellum. J Physiol 470:243-271.

Young SR, Rubel EW (1983) Frequency-specific projections of individual neurons in chick brainstem auditory nuclei. J Neurosci 3:1373-1378.

Young SR, Rubel EW (1986) Embryogenesis of arborization pattern and topography of individual axons in N. laminaris of the chicken brain stem. J Comp Neurol 254:425-459. 